\title{
Reversible high-pressure phase transition in LaN
}

\author{
Sebastian B. Schneider, ${ }^{1}$ Dominik Baumann, ${ }^{1}$ Ashkan Salamat, ${ }^{2}$ and Wolfgang Schnick ${ }^{1, a)}$ \\ ${ }^{1}$ Department Chemie, Ludwig-Maximilians-Universität, 81377 München, Germany \\ ${ }^{2}$ European Synchrotron Radiation Facility (ESRF), 38000 Grenoble, France
}

(Received 16 February 2012; accepted 29 March 2012; published online 1 May 2012)

\begin{abstract}
In situ high-pressure X-ray powder diffraction experiments on LaN up to $60.1 \mathrm{GPa}$ at ambient temperature in a diamond-anvil cell revealed a reversible, first-order structural phase transition starting at $\sim 22.8 \mathrm{GPa}$ and completed at $\sim 26.5 \mathrm{GPa}$ from the ambient cubic phase $(F m \overline{3} m$, no. 225$)$ to a tetragonal high-pressure phase $(P 4 / n m m$, no. $19, a=4.1060(6), c=3.0446(6) \AA, Z=2$, $w R_{\mathrm{p}}=0.011$ ), which has not been claimed in theoretical predictions. HP-LaN is isotypic with a high-pressure polymorph of $\mathrm{BaO}$, which crystallizes in a tetragonally distorted $\mathrm{CsCl}$-type structure. The phase transition is accompanied by a volume collapse of about $11 \%$ which corresponds well with the reported data on HP-BaO. A linear extrapolation of the $c / a$ ratio of the tetragonally distorted CsCl-type sub-cell reaches a value $c / a=1$ of cubic CsCl-type HP-LaN at 91(12) GPa. In addition, the compressibility of LaN was investigated and resulted in a bulk modulus for the ambient pressure phase of $B_{0}=135(3) \mathrm{GPa}$ and $B^{\prime}=5.0(5)$ after fitting a third-order Birch-Murnaghan equation of state to the experimental $p-V$ data. The corresponding extrapolated bulk modulus of HP-LaN is found to be $B_{0}=278(6) \mathrm{GPa}$ and its pressure derivative $B^{\prime}=1.2(2)$. Both as-calculated bulk moduli are compared to the respective values obtained from an Eulerian strain versus normalized stress plot to be 143(2) GPa for ambient LaN and 293(7) GPa for HP-LaN. Compared to other binary nitrides such as $\delta$ - $\mathrm{ZrN}$ or $\delta$ - $\mathrm{HfN}$ having bulk moduli of $285 \mathrm{GPa}$ and $306 \mathrm{GPa}$, respectively, the extrapolated bulk moduli of HP-LaN are in the same order of magnitude, ranking HP-LaN as a highly incompressible material. (C) 2012 American Institute of Physics.
\end{abstract}

[http://dx.doi.org/10.1063/1.4709392]

\section{INTRODUCTION}

Recently, there has been extensive interest in scientific and technological application of rare earth mononitrides amongst which LaN in particular turned out to be very promising due to its unique optical, electronic, magnetic, mechanical, and vibrational properties related to its crystal structure. ${ }^{1-8}$ As the high-temperature and ambient pressure structure for $\mathrm{LaN}$ is the well-known rocksalt structure type (B1) in space group $F m \overline{3} m$ (no. 225), the high-pressure behavior of this compound attracted remarkable interest over the last few years. ${ }^{2,3,6,9-12}$ In order to further study the exceptional properties of LaN at high-pressure conditions, pressure-induced structural phase transitions have been investigated theoretically. Thereby, a phase transition into the CsCl-type structure (B2) has been predicted at transition pressures of 25-100 GPa depending on the applied theoretical method. The corresponding calculated bulk moduli of B1 and the latter B2 phase range in between 121-173 GPa and 88-282 GPa, respectively. However, no experimental data confirming any of the theoretical predictions have been obtained so far. Recently, Hasegawa et al. succeeded in synthesizing two novel lanthanum nitrides with $R E_{2} \mathrm{~N}_{3}$ composition using laser heated diamond-anvil cells. However, they did not start with the mononitride itself, but with elemental lanthanum and nitrogen, the latter acting both as pressure transmitting medium and reacting agent. ${ }^{13}$

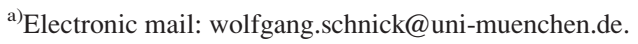

Here, we report on the first experimental in situ highpressure data for LaN obtained with the diamond-anvil cell technique to verify the predicted B1-B2 phase transition. Therefore, the high-pressure structural evolution up to $60.1 \mathrm{GPa}$ is reported. Additionally, the compressibility of LaN and an observed high-pressure polymorph, which is isotypic with a high-pressure phase of $\mathrm{BaO}^{14-19}$ and homeotypic with $\mathrm{Ph} 4 \mathrm{X}(\mathrm{X}=\mathrm{Br}, \mathrm{I}),{ }^{20,21}$ is investigated by a thirdorder Birch-Murnaghan equation of state fitted to the obtained $p-V$ data and compared to the values obtained from an Eulerian strain versus normalized stress $F(f)$-plot and to predicted values in the literature. ${ }^{2,3,6,9-12}$

\section{EXPERIMENTAL DETAILS}

\section{A. In situ x-ray powder diffraction}

In situ X-ray powder diffraction experiments at high pressure were carried out on beamline ID27 at the ESRF. ${ }^{22} \mathrm{~A}$ membrane driven diamond-anvil cell equipped with $250-\mu \mathrm{m}$ culet diamonds and a rhenium gasket was used with an automated pressure drive. ${ }^{23}$ The gasket was pre-indented to $38 \mu \mathrm{m}$ and a $120-\mu \mathrm{m}$ hole working as a sample chamber was drilled into the gasket using a Nd:YAG laser. Nitrogen, $\mathrm{N}_{2}$ (gas loading), served as the pressure transmitting medium (PTM). The sample (LaN, Alfa Aesar, 99.9\%) was loaded onto a bedding of three ruby spheres. As LaN is extremely sensitive to moisture, the entire loading procedure had to be performed in an argon filled glove box. The pressure was determined by the ruby fluorescence scale according to the calibration (Ne) described by Mao et $a l .{ }^{24}$ For the high-pressure experiment, 


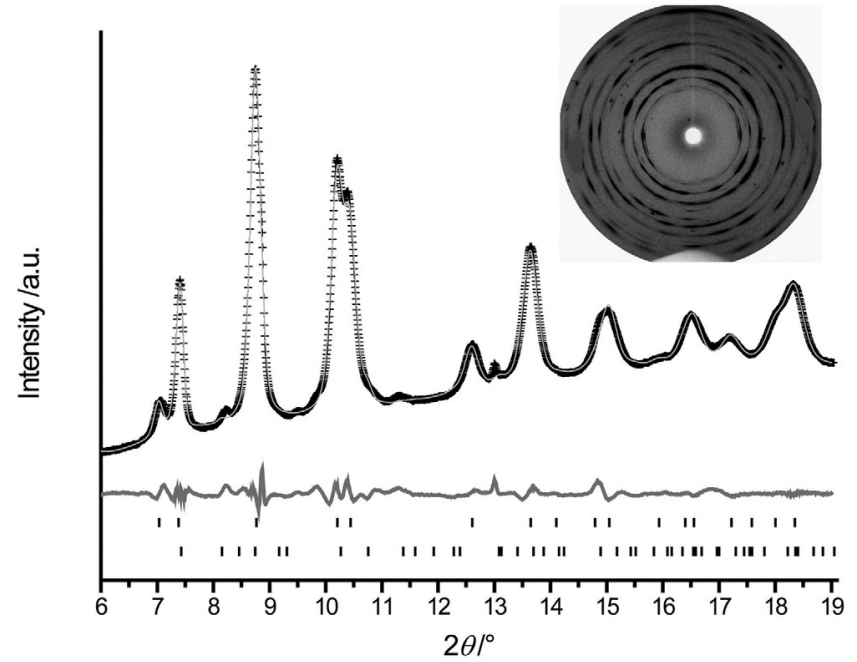

FIG. 1. Observed (crosses) and calculated (gray line) diffraction pattern as well as difference profile of the Rietveld refinement of HP-LaN at $60.1 \mathrm{GPa}$; peak positions of HP-LaN (top) and $\varepsilon-\mathrm{N}_{2}$ (bottom) are marked by vertical lines. Two-dimensional image plate data (upper right) were reduced and corrected (masking of diamond spots, dark gray) using the software FIT2D. monochromatic radiation with a wavelength of $\lambda=0.3738 \AA$ was selected. The beam was focused on a $3 \times 3 \mu \mathrm{m}^{2}$ area and a total number of 60 diffraction images were collected at pressures ranging from 1.3 to $60.1 \mathrm{GPa}$. The pressure at each diffraction image was measured before and after the data collection and averaged to its final value. Diffracted intensities were recorded with a MAR165 charge-coupled device detector. Exposure time was typically 5-20 s, depending on saturation of the detector. To average on texture and strain effects for selected images, the cell was oscillated in general by $\pm 10^{\circ}$ relative to the beam. A precise calibration of the detector parameters was performed with $\mathrm{LaB}_{6}$ powder as reference material. The diffraction images were processed and integrated using the program FIT2D. ${ }^{25}$ Individual single diffraction spots from the diamond anvils were masked manually and excluded from integration.

Indexing and Rietveld refinement for both phases was performed with the TOPAS package. ${ }^{26}$ In case of LaN crystallizing in the B1-type structure, the crystallographic data at ambient conditions ${ }^{27}$ were taken as starting values for stepwise Rietveld refinement up to higher pressures. The crystal structure of

TABLE I. Details of the Rietveld refinements and in situ crystallographic data for ambient LaN and HP-LaN at various pressures.

\begin{tabular}{|c|c|c|c|c|c|}
\hline \multirow{2}{*}{$\begin{array}{l}\text { Compound } \\
\text { Pressure (GPa) }\end{array}$} & & \multicolumn{2}{|c|}{$\mathrm{LaN}$} & \multicolumn{2}{|c|}{ HP-LaN } \\
\hline & & $1.3(5)$ & $19.7(5)$ & $39.9(5)$ & $60.1(5)$ \\
\hline \multicolumn{2}{|l|}{ Structure type } & \multicolumn{2}{|c|}{$\mathrm{NaCl}$} & \multicolumn{2}{|c|}{$\mathrm{HP}-\mathrm{BaO}$, (Refs. 14-19) $\mathrm{PH}_{4} \mathrm{X}(\mathrm{X}=\mathrm{Br}, \mathrm{I})$ (Refs. 20 and 21) } \\
\hline \multirow{2}{*}{\multicolumn{2}{|c|}{$\begin{array}{l}\text { Space group } \\
a(\AA)\end{array}$}} & \multicolumn{2}{|c|}{$F m \overline{3} m($ no. 225$)$} & \multicolumn{2}{|c|}{$P 4 / n m m($ no. 129, origin 1$)$} \\
\hline & & $5.2912(7)$ & $5.1103(10)$ & $4.1875(18)$ & $4.1060(6)$ \\
\hline$c(\AA)$ & & & & $3.1579(10)$ & $3.0446(6)$ \\
\hline$V\left(\AA^{3}\right)$ & & $148.14(6)$ & $133.46(8)$ & $55.37(5)$ & $51.33(1)$ \\
\hline Z & & 4 & 4 & 2 & 2 \\
\hline$\rho\left(\mathrm{g} / \mathrm{cm}^{3}\right)$ & & $6.8557(1)$ & $7.61(0)$ & $9.1702(1)$ & $9.8929(1)$ \\
\hline \multicolumn{6}{|l|}{ Atom (site) } \\
\hline \multirow[t]{3}{*}{ La1 (4b) } & $x$ & $1 / 2$ & $1 / 2$ & & \\
\hline & $y$ & $1 / 2$ & $1 / 2$ & & \\
\hline & $z$ & $1 / 2$ & $1 / 2$ & & \\
\hline \multirow[t]{3}{*}{ N1 (4a) } & $x$ & 0 & 0 & & \\
\hline & $y$ & 0 & 0 & & \\
\hline & $z$ & 0 & 0 & & \\
\hline \multirow[t]{3}{*}{ La1 (2c) } & $x$ & & & 0 & 0 \\
\hline & $y$ & & & $1 / 2$ & $1 / 2$ \\
\hline & $z$ & & & $0.3450(23)$ & $0.3586(8)$ \\
\hline \multirow[t]{3}{*}{ N1 (2a) } & $x$ & & & 0 & 0 \\
\hline & $y$ & & & 0 & 0 \\
\hline & $z$ & & & 0 & 0 \\
\hline $2 \theta$ range $\left({ }^{\circ}\right)$ & & \multicolumn{2}{|c|}{$6-19$} & \multicolumn{2}{|c|}{$6-19$} \\
\hline \multicolumn{2}{|l|}{ Method of refinement } & \multicolumn{2}{|c|}{ Fundamental parameter model } & \multicolumn{2}{|c|}{ Fundamental parameter model } \\
\hline Program package & & \multicolumn{2}{|c|}{ TOPAS ACADEMIC } & \multicolumn{2}{|c|}{ TOPAS ACADEMIC } \\
\hline Atomic parameters & & \multicolumn{2}{|c|}{2} & \multicolumn{2}{|c|}{4} \\
\hline \multirow{2}{*}{\multicolumn{2}{|c|}{$\begin{array}{l}\text { Background function } \\
\text { /parameters }\end{array}$}} & \multicolumn{2}{|c|}{ Shifted Chebyshev } & \multicolumn{2}{|c|}{ Shifted Chebyshev } \\
\hline & & 8 & 8 & 12 & 12 \\
\hline
\end{tabular}


HP-LaN was solved $a b$ initio at $60.1 \mathrm{GPa}$ (see Fig. 1). The indexing resulted unambiguously in a tetragonal unit cell with $a=4.12$ and $c=3.05 \AA$. Integrated intensities were extracted using the Pawley-method. ${ }^{28}$ The structure solution using the charge-flipping algorithm ${ }^{29}$ yielded the complete structural model in space group $P 4 / \mathrm{nmm}^{30}{ }^{30}$ Subsequently, the lattice and atomic parameters were refined using the Rietveld method. The reflection profiles were determined using the fundamental parameters approach ${ }^{31}$ by convolution of appropriate source emission profiles with axial instrument contributions and crystalline microstructure effects. Preferred orientation of the crystallites was described with a spherical harmonics function of 8th order. A structural search within several databases with corresponding lattice parameters in the tetragonal crystal system showed that HP-LaN is isotypic with a high-pressure polymorph of $\mathrm{BaO}$ and homeotypic with $\mathrm{PH}_{4} \mathrm{X}(\mathrm{X}=\mathrm{Br}, \mathrm{I})$ crystallizing in a tetragonally distorted CsCl-type structure. Depending on pressure, the corresponding HP-phases of nitrogen $\left(\delta \text { - and } \varepsilon-\mathrm{N}_{2}\right)^{32,33}$ have also been refined together with ambient or/and HP-LaN in order to obtain the correct lattice parameters upon Rietveld refinement of both LaN phases (see Table I). In between the starting and final pressure of transformation (22.8-26.5 GPa), both LaN phases were refined simultaneously. As the structure of HP-LaN was solved and refined at $60.1 \mathrm{GPa}$, these corresponding values were taken as starting values for the stepwise Rietveld refinement down to lower pressures.

Further information of the crystal structure of HP-LaN at 60.1 GPa (Ref. 33) may be obtained from the Fachinformationszentrum Karlsruhe on quoting the depository number CSD-423936.

\section{B. Equation of state}

The isothermal bulk moduli $B_{0}$ and their pressure derivatives $B^{\prime}$ for both LaN phases were analysed from fits of a third-order Birch-Murnaghan (BM) equation of state (EoS) (Refs. 34 and 35 ) to the obtained $p-V$ data at $300 \mathrm{~K}$ with an error in pressure set to $0.5 \mathrm{GPa}$

$$
p=3 B_{0} f(1+2 f)^{5 / 2} \times\left[1+3 / 2\left(B^{\prime}-4\right) f\right],
$$

where

$$
f=\left[\left(V / V_{0}\right)^{-2 / 3}-1\right] / 2,
$$

represents the Eulerain strain, in which $V_{0}$ reflects the volume at zero pressure, so

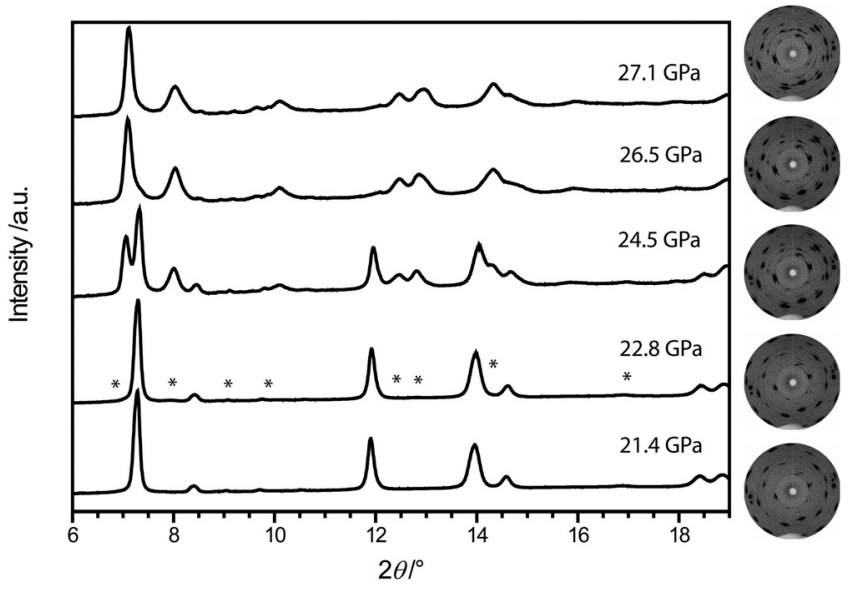

FIG. 2. Selected XRD images and patterns illustrating the phase transition from ambient to HP-LaN starting at $22.8 \mathrm{GPa}$ (asterisks) and completed at $26.5 \mathrm{GPa}$.

$$
\begin{aligned}
p= & 3 / 2 B_{0}\left[\left(V / V_{0}\right)^{-7 / 3}-\left(V / V_{0}\right)^{-5 / 3}\right] \\
& \left.\times\left\{1-3 / 4\left(4-B^{\prime}\right)\left[\left(V / V_{0}\right)^{-2 / 3}-1\right)\right]\right\} .
\end{aligned}
$$

For the ambient phase of LaN, the lattice parameters at zero pressure were taken from the Pearson's Crystal Database. $^{27}$ To obtain the lattice parameters at zero pressure for HP-LaN, the refined parameters at high pressures were extrapolated to zero pressure. Therefore, the bulk modulus and the first pressure derivative of HP-LaN refer to the extrapolated values to zero pressure. To check the quality of the EoS fit, the BM-EoS was also expressed in terms of the Eulerian strain $f$ and the normalized pressure $F$, which is

$$
F=p / 3 f(1+2 f)^{5 / 2} \text {. }
$$

The compression data are therefore additionally presented in a $F(f)$-plot, ${ }^{36}$ where the intercept with the $F$-axis yields $B_{0}$, whereas the slope is $3 \times B_{0}\left(B^{\prime}-4\right) / 2$ and thus provides $B^{\prime}$.

$$
F=B_{0}+\left[3 B_{0}\left(B^{\prime}-4\right) f\right] / 2 .
$$

The $F(f)$ form of representation of the $p-V$ data is very sensitive to scattering of data and to experimental uncertainties as the bulk modulus and the first pressure derivative are obtained by a linear fit. ${ }^{35}$ Both expressions of $B_{0}$ and $B^{\prime}$, the third-order BM-EoS fits and the $F(f)$-plot calculations were done using the software origin. ${ }^{37}$
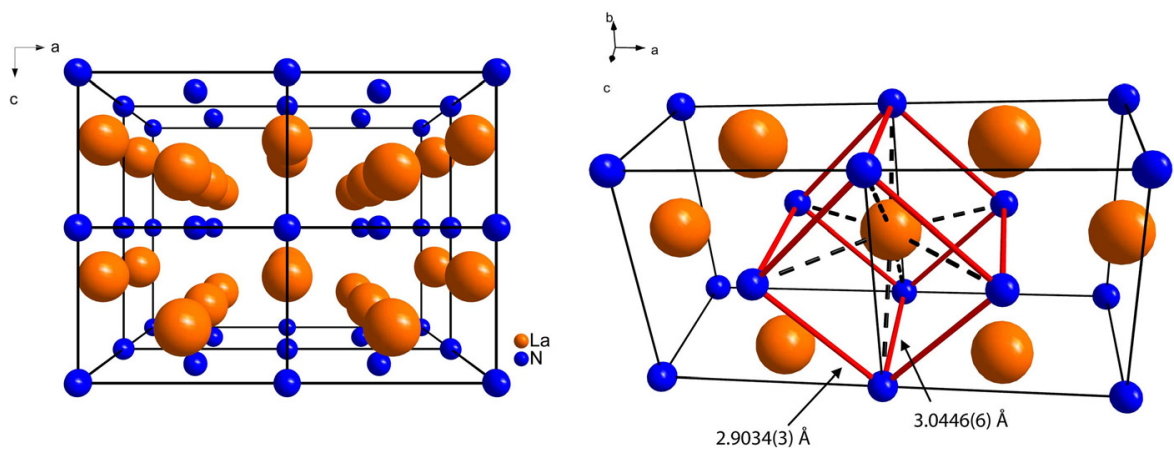

FIG. $3.2 \times 2 \times 2$ super cell of HP-LaN at $60.1 \mathrm{GPa}$ orientated along [101] (left) and doubled unit cell with illustration of the tetragonally distorted sub-cell of the CsCl-type structure indicated by continuous lines between the nitrogen atoms (right). 
(a)

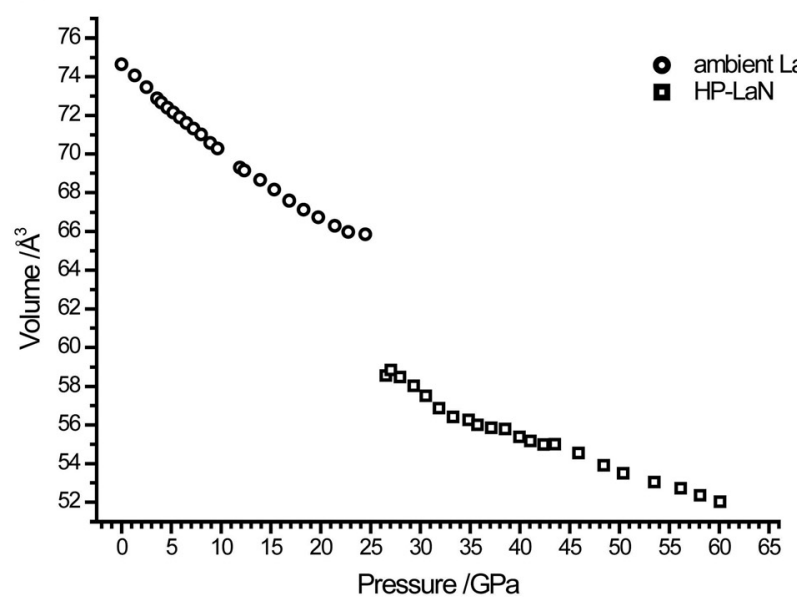

(b)

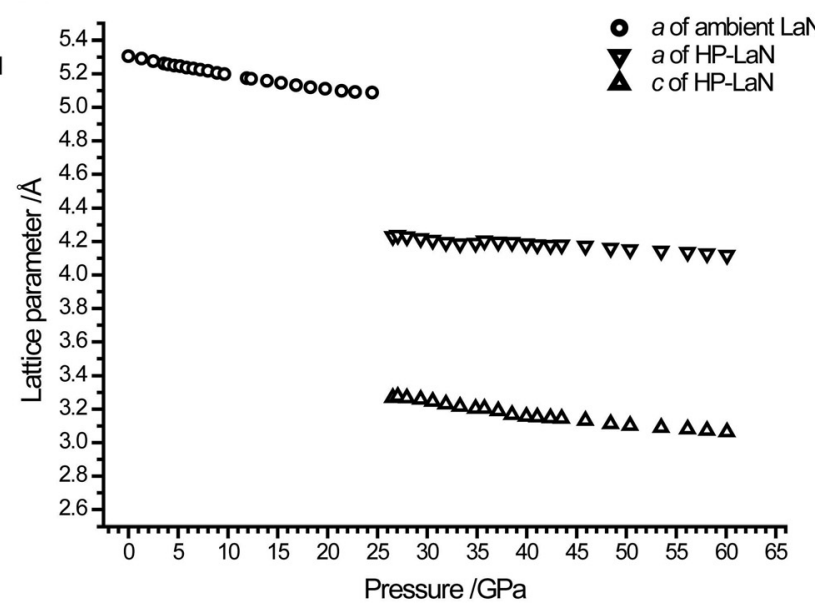

FIG. 4. (a) $V(p)$ plot of ambient and HP-LaN. The volume is given with respect to $Z=2$ to better compare the ambient $(Z=4)$ to the high-pressure phase $(Z=2)$. The volume collapse of about $11 \%$ clearly indicates a first-order phase transition. (b) Evolution of lattice parameters of both LaN phases with pressure.

\section{RESULTS AND DISCUSSION}

\section{A. Phase transition and crystal structure of HP-LaN}

Cubic B1-type LaN starts to transform into a tetragonal high-pressure polymorph at $22.8 \mathrm{GPa}$ (see Fig. 2) in space group $P 4 / \mathrm{nmm}$ (no. 129). The completion pressure of this reversible phase transformation is found to be $26.5 \mathrm{GPa}$. The high-pressure phase of $\mathrm{LaN}$ is isotypic with a high-pressure polymorph of $\mathrm{BaO}$ (stable above $15 \mathrm{GPa}$ ) and homeotypic with $\mathrm{PH}_{4} \mathrm{X}(\mathrm{X}=\mathrm{Br}, \mathrm{I})$ crystallizing in a tetragonally distorted CsCl-type (see Fig. 3). After decompression, ambient $\mathrm{LaN}$ is recovered at $1.0 \mathrm{GPa}$. If the high-pressure behavior of $\mathrm{LaN}$ is compared with the structural sequence of $\mathrm{BaO}$ upon high-pressure, the intermediate NiAs-type structure of $\mathrm{BaO}$
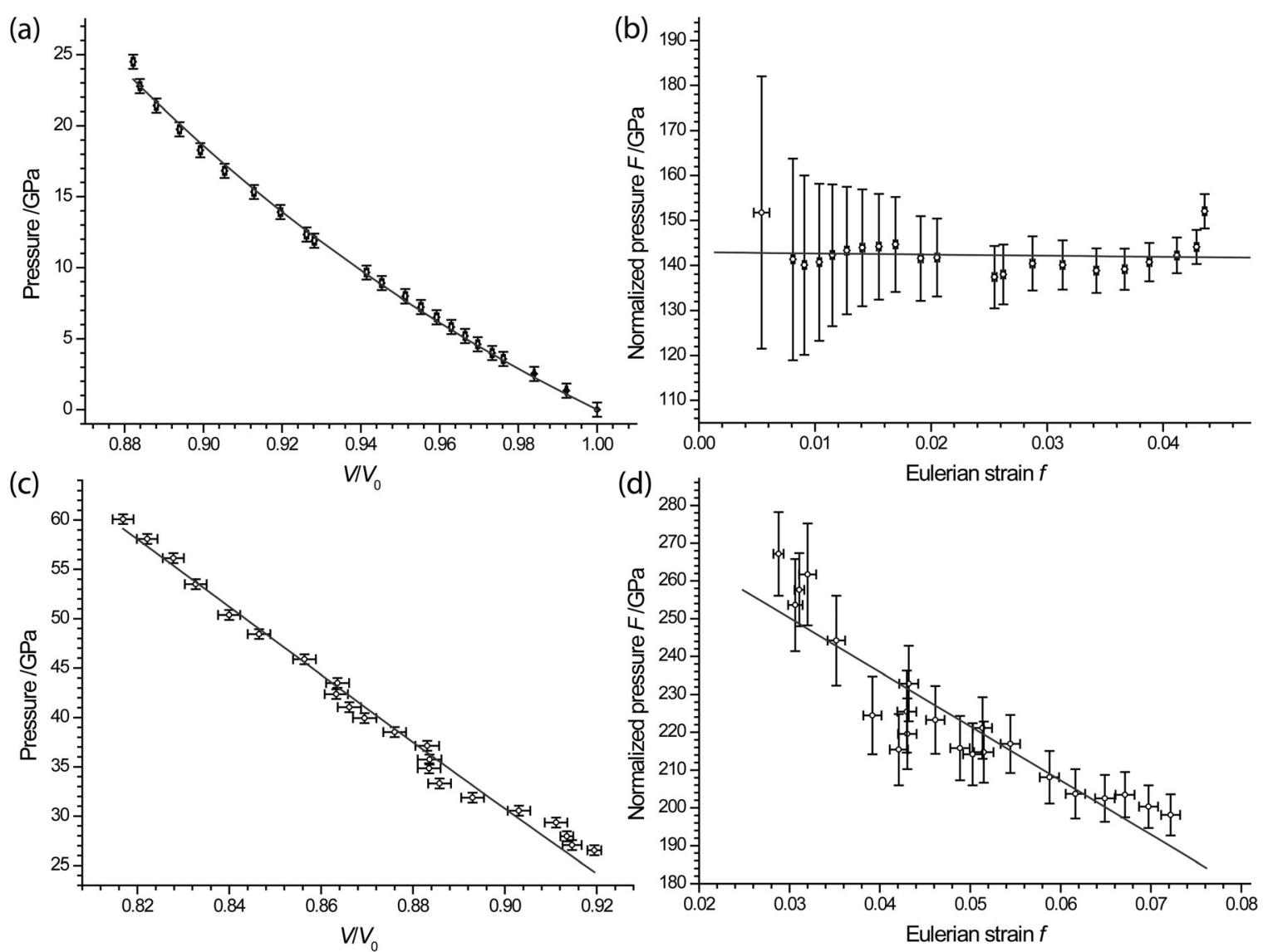

FIG. 5. (a) and (c) Third-order Birch-Murnaghan fits (gray line) to the experimental $p-V$ data (left) of ambient LaN and HP-LaN. For HP-LaN, the volume at zero pressure $\left(V_{0}\right)$ was obtained by extrapolation of the $p-V$ data to ambient conditions and the first $p-V$ data were taken at $26.5 \mathrm{GPa}$. (b) and (d) $F(f)$-plot with linear fits (gray line) to the applied values of the third-order BM-EoS of ambient LaN and HP-LaN. All fits are in fair agreement to the experimental data. The error in pressure is set to $0.5 \mathrm{GPa}$. Top: ambient LaN; bottom: HP-LaN. 
forming at only $10 \mathrm{GPa}$ has not been observed for LaN. ${ }^{14-19}$ Additionally, this structure type has not been mentioned in the theoretical high-pressure predictions of $\mathrm{LaN}^{2,3,6,9-12}$ Accordingly, the B1-type structure transforms directly into the $\mathrm{CsCl}$ structure type at $25-100 \mathrm{GPa}$. For $\mathrm{BaO}$, the $\mathrm{B} 2$ structure is found to be stable at a pressure of $60.5 \mathrm{GPa}$. This might indicate that at a pressure higher than $60.1 \mathrm{GPa}$ another phase transformation from the tetragonally distorted into the cubic $\mathrm{CsCl}$ structure type is observable. This presumption is verified by a linear extrapolation of the $c / a$ ratio of the tetragonally distorted CsCl-type sub-cell (see Fig. 3 continuous lines) which reaches a value $c / a=1$ of cubic CsCl-type HP-LaN at 91(12)GPa.

The crystal structure of HP-LaN is built up of only two formula units per unit cell in contrast to ambient LaN with $Z=4$. According to the (distorted) CsCl-type structure, the coordination number of both ions is 8 resulting in a (distorted) cubic coordination sphere (see Fig. 3).

Upon transformation from ambient cubic to tetragonal $\mathrm{LaN}$, the volume drops by $\sim 11 \%$ (see Fig. 4), clearly indicating a first-order phase transition. The extrapolated unit cell volume of HP-LaN at ambient pressure is smaller by about $14.7 \%$ compared with that of ambient LaN. This is quite reasonable considering the difference in volume at the phase transition.

\section{B. EoS analysis}

Fits of a third-order Birch-Murnaghan equation of state to the experimental $p-V$ data are presented in Figs. 5(a) and $5(\mathrm{c})$. The corresponding linear fits of the $F(f)$-plot are illustrated in Figs. 5(b) and 5(d). To calculate the bulk modulus $B_{0}$ and the first pressure derivative of the high-pressure phase of LaN, the $p-V$ data were extrapolated to zero pressure giving $V_{0}$, which was used as starting volume for the calculations. The results of all fits are summarized in Table II. Since the differences in $B_{0}$ and $B^{\prime}$ obtained from EoS analysis and the linear fit of the $F(f)$-plots are in the same range, the EoS fit can be considered of good quality. In addition, the obtained bulk moduli of ambient LaN do perfectly match the predicted values of $121-173 \mathrm{GPa}^{2,3,6,9-12}$ The extrapolated

TABLE II. Bulk moduli $B_{0}$ and first pressure derivatives $B^{\prime}$ of ambient LaN and HP-LaN calculated by a third-order Birch-Murnaghan EoS and normalized strain fits to the experimental $p-V$ data.

\begin{tabular}{|c|c|c|}
\hline & BM 3rd & Nat. Strain 3rd \\
\hline \multicolumn{3}{|c|}{ Ambient LaN } \\
\hline$a_{0}(\AA)$ & \multicolumn{2}{|c|}{5.305} \\
\hline$V_{0}\left(\AA^{3}\right)$ & \multicolumn{2}{|c|}{149.3} \\
\hline$B_{0}(\mathrm{GPa})$ & $135.5(3.5)$ & $142.9(1.8)$ \\
\hline$B^{\prime}$ & $5.0(5)$ & $3.9(2)$ \\
\hline \multicolumn{3}{|l|}{ HP-LaN } \\
\hline$a_{0}(\AA)$ & \multicolumn{2}{|c|}{$4.3169(30)$} \\
\hline$c_{0}(\AA)$ & \multicolumn{2}{|c|}{$3.4424(31)$} \\
\hline$V_{0}\left(\AA^{3}\right)$ & \multicolumn{2}{|c|}{$63.68(1)$} \\
\hline$B_{0}(\mathrm{GPa})$ & $278.5(6.4)$ & 293.2(7.2) \\
\hline$B^{\prime}$ & $1.2(2)$ & $0.7(2)$ \\
\hline
\end{tabular}

bulk moduli of HP-LaN with values of 278.5(6.4) and 293.2(7.2) GPa are extremely high, ranking HP-LaN as a highly incompressible material comparable to $\delta$ - $\mathrm{ZrN}$ or $\delta$ HfN having bulk moduli of $285 \mathrm{GPa}$ and $306 \mathrm{GPa}$, respectively. ${ }^{38}$

As expected, the high-pressure phase is less compressible than the ambient phase and shows a smaller first pressure derivative of the bulk modulus. This corresponds to the usually expected behavior as the repulsive forces of atoms become stronger at higher pressure. According to the sudden drop in volume at the phase transition, the bulk modulus increases.

\section{CONCLUSION}

By in situ high-pressure experiments on $\mathrm{LaN}$ in a diamond-anvil cell, we were able to identify a reversible, first-order phase transition from the ambient cubic phase to a tetragonally distorted CsCl-type structure, which was not considered in recent theoretical predictions. Therefore, to properly predict the high-pressure behavior of $\mathrm{LaN}$, the high-pressure phase has to be taken into account. HP-LaN is isotypic with a high-pressure polymorph of $\mathrm{BaO}$ undergoing a final phase transition into the B2-type structure at pressures higher than $60 \mathrm{GPa}$. In this context, we experimentally predict another phase transition to the cubic $\mathrm{CsCl}$-type phase at a pressure of 91(12) GPa. Furthermore, this observation matches the theoretical predicted pressure range for a B1-B2 transition. Additionally, the compressibility was analyzed for both phases. For ambient LaN, the obtained bulk moduli perfectly match the predicted values. For HP-LaN, the bulk moduli range in between those of $\delta$ - $\mathrm{ZrN}$ or $\delta$-HfN which ranks HP-LaN a highly incompressible material.

\section{ACKNOWLEDGMENTS}

The authors would like to thank the ESRF for access to their instruments and support during the beamtime. The financial support from the Fonds der Chemischen Industrie (FCI) and the Deutsche Forschungsgemeinschaft (DFG), project SCHN 377/13-2 is gratefully acknowledged.

\footnotetext{
${ }^{1}$ V. A. Gubanov, A. L. Ianovsky, and V. P. Zhukov, in Electronic Structure of Refractory Carbides and Nitrides (Cambridge University Press, Cambridge, 1994).

${ }^{2}$ Y. O. Ciftci, K. Colakoglu, E. Deligoz, and H. Ozisik, Mater. Chem. Phys. 108, 120 (2008).

${ }^{3}$ G. Vaitheeswaran, V. Kanchana, and M. Rajagopalan, Solid State Commun. 124, 97 (2002).

${ }^{4}$ F. Hulliger, J. Magn. Magn. Mater. 8, 183 (1978).

${ }^{5}$ K. A. Gschneider, Jr., and I. R. Eyring, in Handbook of Physics and Chemistry of Rare Earths (North Holland, Amsterdam, 1974), Vol. 4, p. 153.

${ }^{6}$ C. Stampfl, W. Mannstadt, R. Asahi, and A. J. Freeman, Phys. Rev. B 63, 155106 (2001).

${ }^{7}$ R. Norman, H. J. F. Jansen, D. D. Koelling, and A. J. Freeman, Solid State Commun. 52, 739 (1984).

${ }^{8}$ A. Hasegawa, J. Phys. C 13, 6147 (1980).

${ }^{9}$ D. R. Hamann, X. Wu, K. M. Rabe, and D. Vanderbilt, Phys. Rev. B 71, 035117 (2005).

${ }^{10}$ A. Rukmangad, M. Aynyas, and S. P. Sanyal, Indian J. Pure Appl. Phys. 47, 114 (2009).

${ }^{11}$ M. Ghezali, B. Amrani, Y. Cherchab, and N. Sekkal, Mater. Chem. Phys. 112, 774 (2008).

${ }^{12}$ S. D. Gupta, S. K. Gupta, and P. K. Jha, Comput. Mater. Sci. 49, 910 (2010).
} 
${ }^{13}$ M. Hasegawa, K. Nivwa, and T. Yagi, Solid State Commun. 141, 267 (2007).

${ }^{14}$ L.-G. Liu, J. Appl. Phys. 42, 3702 (1971).

${ }^{15}$ L.-G. Liu and W. A. Bassett, J. Geophys. Res. 77, 4934, doi:10.1029/ JB077i026p04934 (1972).

${ }^{16}$ M. Uludoğan, T. Çağin, A. Strachan, and W. A. Goddard III, J. Comput.Aided Mater. Des. 8, 193 (2001).

${ }^{17}$ R. G. Amorim, M. Verissimo-Alves, and J. P. Rino, Comput. Mater. Sci. 37, 349 (2006).

${ }^{18}$ S. T. Weir, Y. K. Vohra, and A. L. Ruoff, Phys. Rev. B: Condens. Matter 33, 4221 (1986).

${ }^{19}$ I. Lukačević, Phys. Status Solidi B 248, 1405 (2011).

${ }^{20}$ A. Sequeira and W. C. Hamilton, J. Chem. Phys. 47, 1818 (1967).

${ }^{21}$ L. W. Schroeder and J. J. Rush, J. Chem. Phys. 54, 1968 (1971).

${ }^{22}$ M. Mezouar et al., J. Synchrotron Radiat. 12, 659 (2005).

${ }^{23}$ R. Letoullec, J. P. Pinceaux, and P. Loubeyre, High Press. Res. 1, 77 (1988).

${ }^{24}$ H.-K. Mao, J. Xu, and M. J. Bell, Geophys. Res. 91, 4673, doi:10.1029/ JB091iB05p04673 (1986).

${ }^{25}$ A. P. Hammersley, S. O. Svensson, M. Hanfland, A. N. Fitch, and D. Hausermann, High Press. Res. 14, 235 (1996).

${ }^{26}$ A.A. Coelho, TOPAS-Academic, Version 4.1, Coelho Software, Brisbane, 2007.

${ }^{27}$ P. Villars, and K. Cenzual, Pearson's Crystal Data-Crystal Structure Database for Inorganic Compounds, Version 1.3d (ASM International, Ohio, 2009/2010).

${ }^{28}$ G. S. Pawley, J. Appl. Crystallogr. 14, 357 (1981).
${ }^{29}$ G. Oszlányi and A. Sütő, Acta Crystallogr., Sect. A: Found. Crystallogr. 60, 134 (2004); G. Oszlányi and A. Sütő, ibid. 64, 123 (2008); A. A. Coelho, ibid. 63, 400 (2007).

${ }^{30}$ Crystal data for HP-LaN at $60.1 \mathrm{GPa}$ : formula: $\mathrm{LaN}, \mathrm{M}=152.92 \mathrm{~g}$ $\mathrm{mol}^{-1}$, space group $P 4 / \mathrm{nmm}$ (no. 129, origin1), $a=4.1060(6)$, $c=3.0446(6) \AA, \quad V=51.33(1) \AA^{3}, \quad \mathrm{Z}=2$, radiation: $\lambda=0.3738 \AA$, $\mathrm{T}=298(2) \mathrm{K}, 17$ reflections, 17 parameters, $\mathrm{R}_{\mathrm{p}}=0.0080, \mathrm{wR}_{\mathrm{p}}=0.011$, $\chi^{2}=1.182, \mathrm{R}_{\text {bragg }}=0.0768$, background: shifted Chebychev, 12 background parameters. Further information of the crystal structure of HP$\mathrm{LaN}$ at $60.1 \mathrm{GPa}$ can be obtained from Fachinformationszentrum Karlsruhe, 76344 Eggenstein-Leopoldshafen, Germany (fax: (49) 7247-808-666; e-mail: crysdata@fiz-karlsruhe.de).

${ }^{31}$ J. Bergmann, R. Kleeberg, A. Haase, and B. Breidenstein, Mater. Sci, Forum 347-349, 303 (2000).

${ }^{32}$ E. Gregoryanz, A. F. Goncharov, C. Sanloup, M. Somayazulu, H.-K. Mao, and R. J. Hemley, J. Chem. Phys. 126, 184505 (2007).

${ }^{33}$ D. T. Cromer, R. L. Mills, D. Schiferl, and L. A. Schwalbe, Acta Crystallogr. B 37, 8 (1981).

${ }^{34}$ F. Birch, Phys. Rev. 71, 809 (1947).

${ }^{35}$ F. Murnaghan, Proc. Natl. Acad. Sci. USA 30, 244 (1944).

${ }^{36}$ D. L. Heinz and R. Jeanloz, J. Appl. Phys. 55, 885 (1984).

${ }^{37}$ Origin 6.1, v6.1052 (B232), OriginLab Corporation, 2000.

${ }^{38}$ X.-J. Chen, V. V. Struzhkin, Z. Wu, M. Somayazulu, J. Qian, S. Kung, A. N. Christensen, Y. Zhao, R. E. Cohen, H.-K. Mao, and R. J. Hemley, Proc. Natl. Acad. Sci. U.S.A. 102, 3198 (2005). 